Original Research Paper

\title{
Total Conductivity of Calcium Iron Borate Glasses Using an Empirical Formula
}

\author{
${ }^{1}$ Hossam M. Gomaa, ${ }^{2}$ Saeid M. Elkatlawy and ${ }^{3}$ Ahmed H. El-Dosokey \\ ${ }^{1}$ Optical Branch, High Institute of Optics Technology, Cairo, Egypt \\ ${ }^{2}$ Department of Physics, Faculty of Science, Damanhour University, Damanhour, Egypt \\ ${ }^{3}$ Department of Physics, Faculty of Science, El-Fayoum University, El-Fayoum, Egypt
}

Article history

Received: 02-03-2019

Revised: $13-05-2019$

Accepted: 20-05-2019

Corresponding Author: Dr. Saeid M. Elkatlawy Department of Physics, Faculty of Science, Damanhour

University, Egypt

Email: saeid.elkatlawy@gmail.com

\begin{abstract}
Samples of calcium borate glasses containing iron ions have been prepared with three different concentrations of both boron and iron oxides. X-ray diffraction spectroscopy was performed and results revealed that samples are in good glassy state, with high homogeneity and short range ordered structure. Electrical conductivity was experimentally measured in the temperature range from 300 to $553 \mathrm{~K}$. Conductivity results have been studied as a function of the static dielectric constant $(\varepsilon)$ and the charge carrier's relaxation time $(\tau)$. The results were modeled by means of an empirical formula that fits the experimental data as a function of both the static dielectric constant and the electric activation energy $\Delta \mathrm{E}$; in addition to the exponent factor $\mathrm{s}$. Total measured conductivity modeled using the proposed empirical formula fits neatly the correlated barrier hopping model.
\end{abstract}

Keywords: Oxide Glass, Electrical Conductivity, Static Dielectric Constant, CBH Model, Empirical Formula

\section{Introduction}

Iron doped calcium/borate glasses are highly important due to their higher thermal expansion coefficients, lower transition temperature and higher electrical conductivities. Borate oxide glasses were utilized for many useful applications, such as solid state batteries and photonics and laser technology (Veerabhadra et al., 2006). The structure of most oxide glasses is more homogeneous when one or more transition metal oxide is added to their chemical composition. Moreover, these glasses are valuable due to their semiconducting behavior and their capability to integrate their structure with Transition Metal Ions (TMI), so that they can coexist in more than one valance state. For instance, in case of $\mathrm{Fe}^{2+}$ and $\mathrm{Fe}^{3+}$, the conduction mechanism can take place by transferring electrons from low to high valence states (Bishay and Makar, 1969; Gawish and Saleh, 1976). In most oxide glasses, electrical conductivity due to ionic motion results from the transport of cations. Studies on iron doped oxide glasses showed that iron ions exist mainly in $\mathrm{Fe}^{3+}$ state, occupy tetrahedral positions and when introduced in higher concentrations it increases the electrical conductivity of the glass (Veerabhadra et al., 2006). On the other hand, it is well known that electrical $a c$ conductivity can shed a considerable light on the nature of conducting processes and mechanisms in oxide glasses doped with ionic conductors (Lestanguennec and Elliott, 1994). The electrical conductivity is considered as the most important property of the transition metal ions doped glasses. The $a c$ conductivity $\sigma_{a c}$ of such glasses is commonly calculated by Jonscher's universal power law $\sigma_{a c}=A \omega^{s}$, where, $A$ is the pre-factor constant, which is a function of temperature and composition, $\omega$ is the angular frequency and $s$ is the exponent factor of the power law, $0 \geq s \geq 1$, (Das et al., 2013; Kumar, 2013; Veerabhadra et al., 2006). In most cases, the separation between the $d c$ and $a c$ conductivities is a very difficult process, so this work presents an empirical expression to describe the measured conductivity as a function of the static dielectric constant, the activation energy and the exponent factor $s$. Then the total measured conductivity can easily be modeled and fitted to the experimentally measured data.

\section{Experimental Work}

Three chemical compositions were chosen to prepare three glass samples, using fast quenching method, according to the chemical formula: $(65-\mathrm{x}) \mathrm{mole} \% \mathrm{~B}_{2} \mathrm{O}_{3}$ $(\mathrm{x}+15)$ mole $\% \mathrm{Fe}_{2} \mathrm{O}_{3}-20$ mole $\% \mathrm{Ca} \mathrm{O}$, where $\mathrm{x}=0,5$ and 10 mole\%. All chemical batches were melted in an electric furnace at $900 \pm 50^{\circ} \mathrm{C}$, for $90 \mathrm{~min}$, then the melt of each 
sample was poured in the air at room temperature. Philips analytical X-Ray diffraction system, type PW3710 with $\mathrm{Cu}$ tube anode, with $\mathrm{K} \alpha$ radiation $(1.54 \AA$ ), was used for XRD analysis. XRD measurements were performed at slow and continuous rate with step size of $1 \%$ min over an angular range of $20-100^{\circ} 2 \theta$. Whereas, electric measurements were carried out in temperature range from 300 to $553 \mathrm{~K}$ by using a computer controlled Stanford LCR bridge model SR 720 with only available four fixed frequencies $0.12,1,10$ and $100 \mathrm{kHz}$. Total conductivity tests were repeated several times for each sample and statistical evaluation of the experimental results showed no significant differences between them. The experimental results were then modeled using the proposed empirical formula and the data fitted to the experimental results.

\section{Results and Discussion}

\section{XRD Patterns}

Figure 1 shows the XRD patterns for powdered glass samples with different $\mathrm{Fe}_{2} \mathrm{O}_{3}$ content, for $\mathrm{x}=0$ and $\mathrm{x}=10$ mole $\%$. Only one broad peak can be observed at about $2 \theta$ $=43^{\circ}$, without any sharp peaks, the thing that refers to the randomness of the internal structure for these two samples. The sample of $\mathrm{x}=5 \mathrm{~mole} \%$ was expected to have same characteristic. Inspecting Fig. 1 more carefully and referring to the XRD database, it can be stated that the observed hump may be due to the diffraction of X-rays from iron captions.

\section{Electrical Conductivity}

Total conductivity measurements were carried out for the studied glasses, at four different frequencies $(0.12,1$, 10 and $100 \mathrm{kHz}$ ) and in temperature range from 300 to 553 K. Samples were homogenous disc shaped with diameter of $6 \mathrm{~mm}$ and thickness of $1.25 \mathrm{~mm}$, coated with silver paste in order to improve the connectivity between samples and electrodes. The LCR Bridge employed in this study is available only with the four frequencies mentioned previously. As seen in Figs. 2, 3 and 4 the measured conductivity shows different thermally activated process for each frequency. Such behavior may be similar to that of semiconducting materials (Abdel-Wahid et al., 2017; Ahmed and AbdelWahid, 2014; Fares, 2011). The electrical conduction can be attributed to the electronic hopping between the two oxidation states of iron ions Fe (III) and Fe (II) (Elkholy and El-Mallawany, 1995; Salman et al., 2004). The experimentally measured conductivities for oxide glasses were studied by many theoretical models (Elliott, 1987). In several cases these models are complicated and difficult to treat the experimental data.

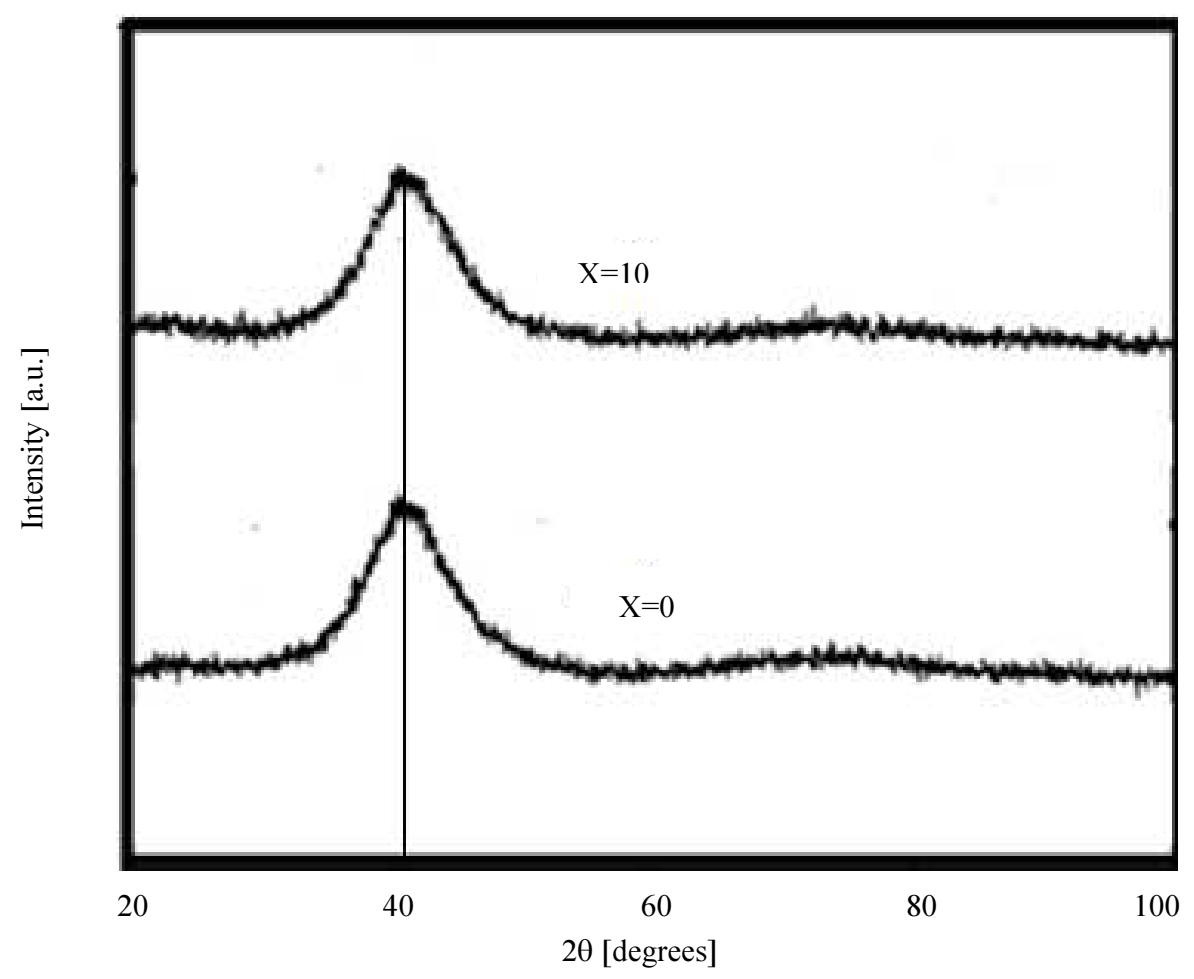

Fig. 1: XRD diffractogram for $\mathrm{x}=0$ and $\mathrm{x}=10$ mole $\%$ samples 


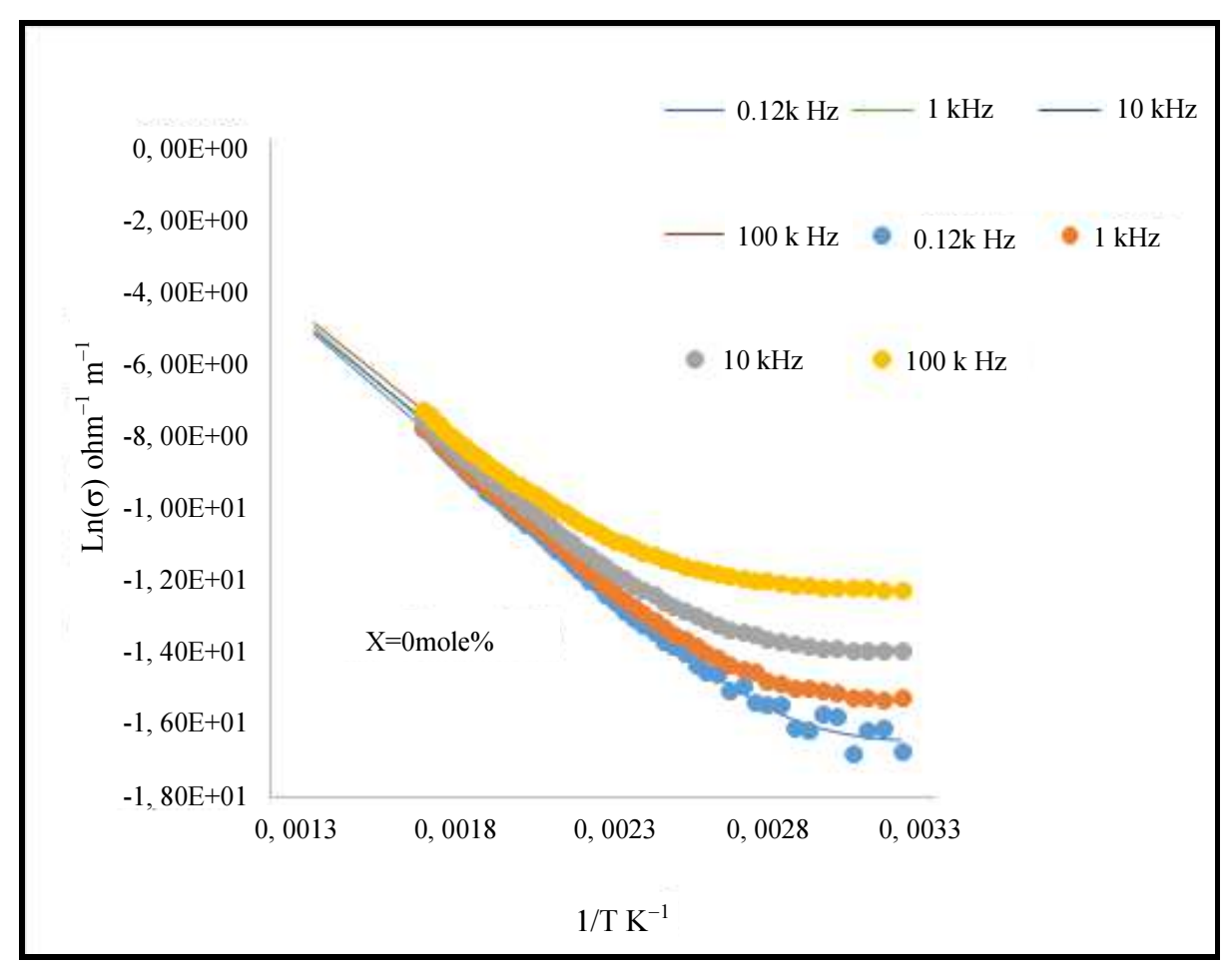

Fig. 2: Conductivity versus the absolute temperature for $x=0$ sample

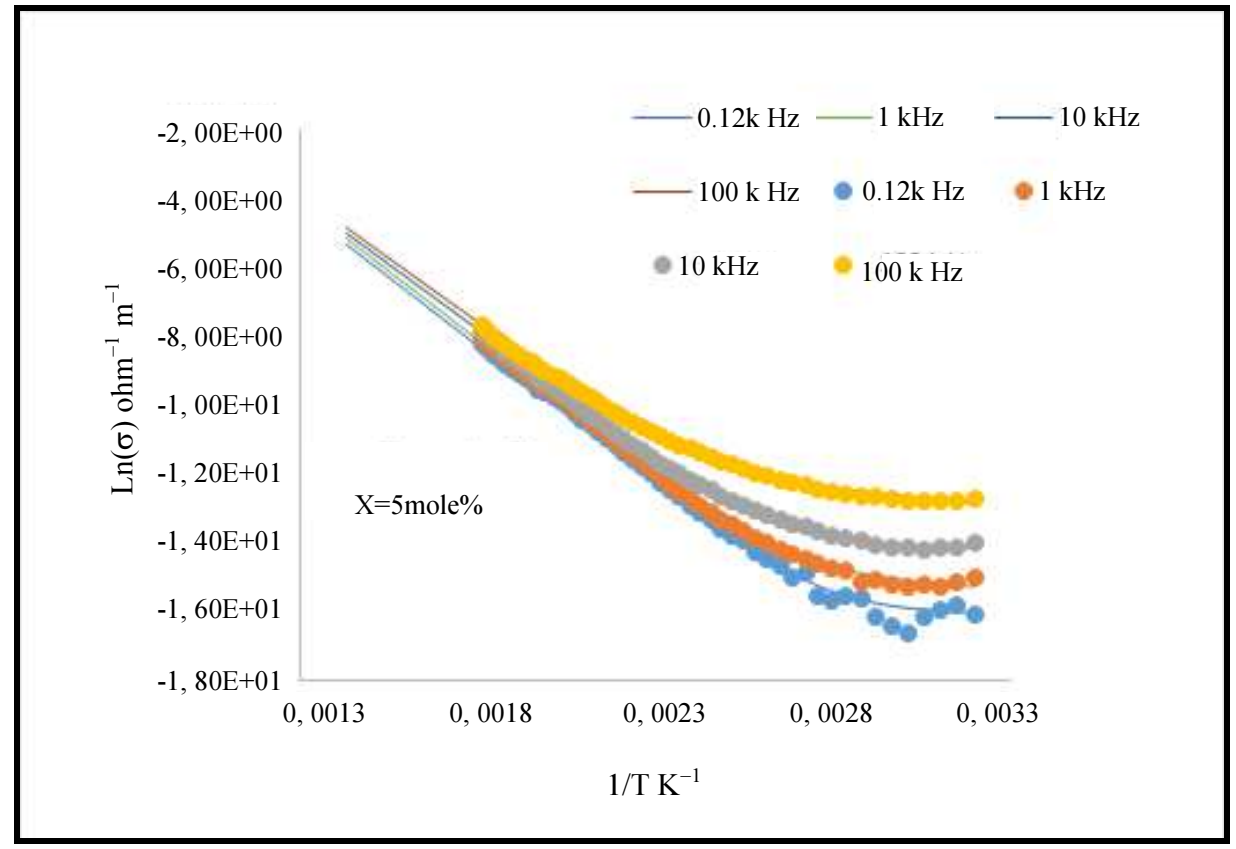

Fig. 3: Conductivity versus the absolute temperature for $x=5$ sample

\section{Empirical Model}

According to the band theory of solids the electrons in the outer most energy levels are practically free to move in all substances irrespective of whether they are metals, semiconductors or insulators. Such movement of electrons cannot induce electric current because of the asymmetrically electrons velocity distribution. In case of 
semiconductors there exists an energy gap between the valance and the conduction bands, the thing which requires not only an external electric field but needs also activation energy to excite the electrons from the valance band to the conduction band. The activation energy may cause some states to split off from the band to form more localized states. But in general, the electrical behavior of the matter depends on its nature, structure, temperature, dimensions, type, origin and the number of charge carriers. In the case of disordered solids (glasses) the electric conduction depends on frequency of the applied electric field.

In any experimental work, the measured conductivity is the sum of the ac and the dc conductivities according to the equation:

$\sigma_{\text {total }}=\sigma(\omega)+\sigma_{d c}$

Each dielectric solid (nonconductors) has a complex relative permittivity $\varepsilon(\omega)$ expressed as a function of the dielectric susceptibility $\chi(\omega)$ as follows:

$$
\varepsilon(\omega)=\varepsilon^{\prime}(\omega)-j \varepsilon^{\prime \prime}(\omega)=\left\{1+\chi^{\prime}(\omega)\right\}-j \chi^{\prime \prime}(\omega)
$$

Accordingly, the electrical conductivity may consist of a real part and an imaginary part. Such that the real part represents the $a c$ conductivity, Eqn. 3, while the imaginary part represents the $d c$ conductivity, Eqn. 4 , as follows:

$$
\begin{aligned}
& \sigma(\omega)=\varepsilon_{0} \omega \varepsilon^{\prime \prime}(\omega) \\
& \sigma_{d c}=\varepsilon_{0} \omega \varepsilon^{\prime}(\omega)
\end{aligned}
$$

Many different micro theoretical models have been applied to explain the $a c$ and $d c$ conductivities, individually, in non-crystalline solids. These models were based on two distinct processes that have been proposed for the relaxation mechanism. These two processes are the mechanical tunneling and classical hopping over potential barrier or a combination of them. In this work authors developed the following empirical formula that fits the experimental data for total measured conductivity, ( $a c$ and $d c$ ), as a function of both the static dielectric constant $\varepsilon$, exponent factor $s$, the electric activation energy $\Delta \mathrm{E}$ and the absolute temperature $T$ multiplied by Boltzmann's constant $k$.

$\sigma_{\text {measured }}=10^{2} \varepsilon e^{\frac{-\Delta E}{k T}}+\omega^{s+1} e^{-41.1 \Delta E}$

Equation 5 represents the empirical formula, the scope of the present work, which was formulated and performed after clearly inspecting to most of macro and micro theoretical models for both $a c$ and $d c$ conductivities. The last investigated formula was used to fit the total measured conductivity for the studied glasses, as seen in Figs. 2, 3 and 4. Where, dots and solid lines represent the experimental and calculated data, respectively. It is clear that the calculated and experimental data are correlated to each other perfectly.

Table (1) contains the calculated values of both $\varepsilon$ and $\Delta \mathrm{E}$ for the studied samples.

\section{Conduction Mechanism}

To characterize the conduction mechanism of the studied glasses, $\operatorname{Ln}(\sigma)$ versus $\operatorname{Ln}(\omega)$ graph was plotted at constant temperature. The slope of the straight part of the $\operatorname{Ln}(\sigma) \sim \operatorname{Ln}(\omega)$ curve gives the value of the exponent factor $\mathrm{s}$ at this temperature:

$s=\frac{d[\operatorname{Ln}(\sigma)]}{d[\operatorname{Ln}(\omega)]}$

The value of the exponent factor $s$ was obtained at each temperature in the range of measurements and then plotted in Fig. 5 as a function of the absolute temperature. By inspecting Fig. 5, it was observed that, the exponent factor decreases as the absolute temperature increases. Such behavior is in agreement with that predicted by the Correlated Barrier Hopping (CBH) model (Elliott, 1987). In other words, the hopping of charge carriers over the potential barrier may be considered as the conduction mechanism for the studied glasses (Ahlawat et al., 2016; Filipic et al., 2012; Pal et al., 2011).

Table 1: The activation energy $\Delta \mathrm{E}$ and the static dielectric constant $\varepsilon$

\begin{tabular}{rrccr}
\hline & $\mathrm{f}(\mathrm{kHz})$ & $\mathrm{x}=0$ & $\mathrm{x}=5$ & $\mathrm{x}=10$ \\
\hline \multirow{4}{*}{$\Delta \mathrm{E}(\mathrm{eV})$} & 0.120 & 0.800 & 0.7050 & 0.70 \\
& 1.000 & 0.800 & 0.7050 & 0.70 \\
& 10.000 & 0.750 & 0.6700 & 0.65 \\
& 100.000 & 0.750 & 0.6700 & 0.65 \\
$\varepsilon$ & 0.120 & 3.500 & 3.5000 & 3.50 \\
& 1.000 & 0.500 & 0.5000 & 0.50 \\
& 10.000 & 0.020 & 0.0330 & 0.02 \\
& 100.000 & 0.002 & 0.0033 & 0.25 \\
\hline
\end{tabular}




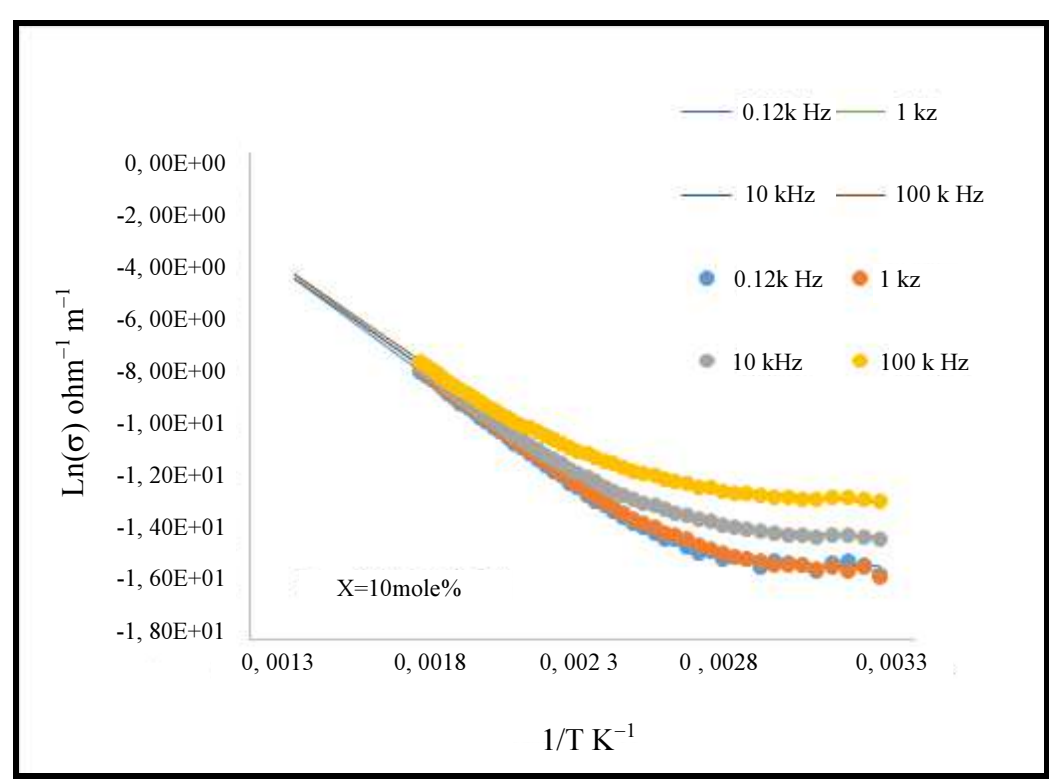

Fig. 4: Conductivity versus the absolute temperature for $\mathrm{x}=10$ sample

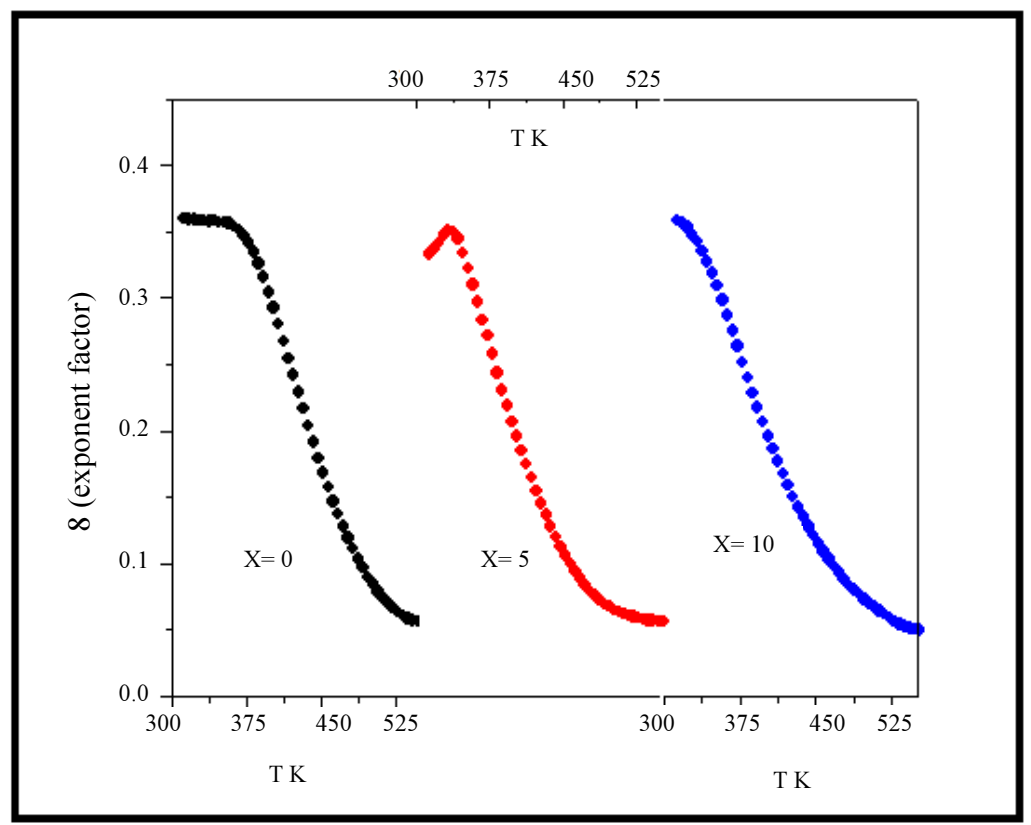

Fig. 5: Power low exponent versus the absolute temperature, for all samples

\section{Conclusion}

An empirical formula was concluded and used to fit and describe the measured data of electric conductivity. For all samples, both the electric activation energy and the static dielectric constant were obtained from the fitting processes. The data analyzed by the empirical formula can fit neatly the experimental data. This signifies the potential of this formula in assessing and analyzing data of $a c$ conductivity of oxide glasses. For all samples, both the electric activation energy and the static dielectric constant were obtained from the fitting processes. The electric conduction mechanism for the studied samples was shown to be following the correlated barrier hopping model.

\section{Acknowledgement}

Authors would like to thank Prof. A. Gamal-Eldin Mostafa and Prof. M. Youssry Hassaan for all their support and encouragement. 


\section{Author's Contributions}

All authors equally contributed in this work.

\section{Ethics}

This article is original and contains unpublished material. The corresponding author confirms that all of the other authors have read and approved the manuscript and there are no ethical issues involved.

\section{References}

Abdel-Wahab, F., A. Maringa and A.A. Monastery, 2017. Electrical conductivity mechanism in unconventional lead vanadate glasses. J. Low Temp. Phys., 186: 372-384.

DOI: $10.1007 / \mathrm{S} 10909-016-1724-4$

Ahlawat, N., P. Aghamkar, A. Agarwal and N. Ahlawat, 2016. Study of conduction mechanism in $\mathrm{Fe}_{2} \mathrm{O}_{3}$ doped $\mathrm{Na}_{2} \mathrm{O} \cdot \mathrm{Bi}_{2} \mathrm{O}_{3} \cdot \mathrm{B}_{2} \mathrm{O}_{3}$ semiconducting glasses. Phys. Condensed Matter, 482: 58-64.

DOI: 10.1016/J.PHYSB.2015.12.014

Ahmed, E.M. and F. Abdel-Wahid, 2014. Unified conduction mechanism in unconventional VZnCaFeO glasses. Phys. Condensed Matter, 449: 246-250. DOI: 10.1016/J.PHYSB.2014.05.046

Bishay, A.M. and L. Makar, 1969. Role of Iron in Calcium Phosphate Glasses. J. Am. Ceramic Soc., 52: 605-609. DOI: 10.1111/J.1151-2916.1969.TB15849.X

Das, P.R., B.N. Parida, R. Padhee and R.N.P. Choudhary, 2013. Electrical properties of $\mathrm{Na}_{2} \mathrm{~Pb}_{2} \mathrm{R}_{2} \mathrm{~W}_{2} \mathrm{Ti}_{4} \mathrm{~V}_{4} \mathrm{O}_{30}(\mathrm{R}=\mathrm{Dy}, \mathrm{Pr})$ ceramics. J. Advan. Ceramics, 2: 112-118. DOI: $10.1007 / \mathrm{S} 40145-013-0048-\mathrm{Y}$

Elkholy, M.M. and R.A. El-Mallawany, 1995. AC. conductivity of tellurite glasses. Materials Chemi. Phys., 40: 163-167.

DOI: $10.1016 / 0254-0584(95) 01472-1$
Elliott, S.R., 1987. Ac conduction in amorphous chalcogenide and pnictide semiconductors. Adv. Phys., 36: 135-217. DOI: 10.1080/00018738700101971

Fares, S., 2011. Frequency dependence of the electrical conductivity and dielectric constants of polycarbonate (Makrofol-E) film under the effects of $\gamma$-radiation. Natural Sci., 03: 1034-1039. DOI: $10.4236 / \mathrm{NS} .2011 .312129$

Filipic, C., A. Moguš-Milanković, L. Pavić, M. Karabulut and A. Levstik, 2012. Polarons in boron doped iron phosphate glasses. J. Non-Crystalline Solids, 358: 2793-2795.

DOI: 10.1016/J.JNONCRYSOL.2012.07.011

Gawish, M. and M.N. Saleh, 1976. Electronic properties of calcium borate glasses containing iron oxide. J. Applied Phys., 47: 5349-5355.

DOI: $10.1063 / 1.322559$

Kumar, T.V., 2013. Dielectric relaxation, ionic conduction and complex impedance studies on $\mathrm{nano}_{3}$ fast ion conductor. Int. J. Materials Sci. Appl., 2: 173. DOI: 10.11648/J.IJMSA.20130206.12

Lestanguennec, M. and S. Elliott, 1994. Frequencydependent ionic conductivity in AgI-AgPO3 glasses. Solid State Ionics, 73: 199-208. DOI: 10.1016/0167-2738(94)90035-3

Pal, M., B. Roy and M. Pal, 2011. Structural characterization of borate glasses containing zinc and manganese oxides. J. Mod. Phys., 02: 1062-1066. DOI: 10.4236/JMP.2011.29129

Salman, F., S. Aboelhssan, E. Sheha and M.K. Elmansy, 2004. Dielectric properties and conductivity of $\mathrm{KHCO}_{3}$. Turkish. J. Phys., 28: 57-63.

Veerabhadra Rao, A., C. Laxmikanth, B. Appa Rao and N. Veeraiah, 2006. Dielectric relaxation and $\mathrm{AC}$ conduction phenomena of $\mathrm{PbO}-\mathrm{PbF}_{2}-\mathrm{B}_{2} \mathrm{O}_{3}$ glasses doped with FeO. J. Phys. Chem. Solids, 67: 22632274. DOI: 10.1016/J.JPCS.2006.04.012 